\title{
Face Recognition by Radial Basis Function Network (RBFN)
}

\author{
Mrinal Kanti Dhar \\ Lecturer, Dept. of EEE \\ Leading University \\ Bangladesh
}

\author{
Quazi M. Hasibul Haque \\ $R$ \& D Engineer, \\ Television Department. \\ Walton Bangladesh
}

\author{
Md. Tanjimuddin \\ Lecturer, Dept. of EEE \\ Leading University \\ Bangladesh
}

\begin{abstract}
Face recognition technology using Radial Basis Function Network (RBFN) is an attractive solution for the researchers who are working on the field of machine recognition, pattern recognition and computer vision. The key challenge in the face recognition technology is to provide high recognition rate. In this paper, an efficient method has been presented for face recognition using principal component analysis and radial basis function. More specifically, principal component analysis has been used for feature extraction and radial basis function network has been used as a classifier to classify data as well as for recognition process.
\end{abstract}

\section{Keywords}

Face recognition, Principal component analysis, Artificial neural network, Radial basis function network.

\section{INTRODUCTION}

In the field of image processing, pattern recognition, computer vision, and neural network, every researcher concentrates on human face recognition by machine from still and video images. For doing this task, researchers are facing some difficulties when real time identification is required. One of the difficulties is that face image is highly variable and another one is variability of sources [1]. Total face recognition is obtained by three steps. These steps are - preprocessing, feature extraction and finally classification and recognition [2].

In the first step of preprocessing step, reduction of noise, possible convolute effects of interfering system are carried out [2]. Next step is feature extraction that plays an important role to represent a face in face recognition system. Feature extraction is one of the special forms of dimensionality reduction. In feature extraction process, input data is transferred into a set of features i.e. the relevant information of the input which will be used to do the desired task [3]. In feature extraction process, many researchers have proposed different techniques, as feature extraction by principal component analysis, independent component analysis, and linear discriminant analysis [4]. One of the most successful techniques among them is principal component analysis for extracting feature and representing data [10]. It not only reduces the dimensionality of the image but also retains some of the variations in the image data and provides compact representation of a face image [4][6]. Principal component analysis (PCA) is a statistical tool to convert a set of observations of possibly co-related variable into a set of values of uncorrelated variable called Principal Component (PC) by using orthogonal transformation [6]. Here,the number of original variables is greater than or equal to the number of principal components. This transformation is defined in such a way that the dataset is placed into a new ordinate system where the variance is put in order from the greatest to the least. That means the first principle component has as high in variance as possible. As the variance is ordered in a decreasing manner, much of the variance or information in the original set is concentrated in the first few PCs. Therefore, the last few PCs can be rejected without losing much information. When data set is jointly normally distributed, it is guaranteed that the principal component is independent. In the time of relative scaling of original variables, PCA is easily affected. The principal component of the PCA is Eigen faces [12]. It is found from the transformation of the face images into a smaller set of characteristics of feature images [6]. Third process is classification and recognition. Classifier analyzes the numerical properties of various images and organizes data into categorized data and then all the recognition processes are done. In recognition process, various classification techniques have been proposed such as radial basis function, back propagation etc. Due to its fast learning ability and computational simplicity, radial basis function has become a good choice for classification. From different types of neural network architectures, Radial Basis Function Network (RBFN) is getting more attention to the researchers for its attractive advantages such as it usually trains much faster than other networks. In addition, they are less susceptible to problems with non-stationery inputs of the behavior of the radial basis function hidden units [5], [11]. Powell was the initiator of the radial basis function by solving the real multivariate interpolation problem [7]. In the field of neural network, radial basis function is first used by Broomhead and Lowe [8]. RBF differs from the perception type networks as its activation of hidden unit depends on the distance between the input vector and a prototype vector, where as the activation of hidden unit in perception type networks is based on the dot product between the input vector and a weight vector. In a high dimensional space, radial basis function is the main gateway by viewing the design as a curve-fitting problem. Finding multidimensional function, which provides the best fit, is the main algorithm of radial basis function [9]. From this angle of vision RBF is getting more and more popularity to the modern researchers in the field of Image processing, Speech processing, Time series analysis adaptive equalization, Radar point source location, Process fault detection and Pattern recognition [8]. In this paper, improvisation of the face recognition process has been done by combining a statistical based method PCA and a neural network based method RBF. PCA method is used for face feature extraction after reducing dimensionality of input space. Structural image information is to be considered for classification process for achieving high recognition rate. This has been realized by identifying sub clusters corresponding to a subject separately using a clustering algorithm. Then the prototypes of these sub clusters are to model the hidden layer 
neurons of the RBF neural network. This process also improves its generalization capabilities.

\section{Experiments on the AT \& T Database}

The AT\&T database (formerly 'The ORL Database of Faces') contains 400 grayscale images of 40 persons. Each person has 10 images. The size of each image is $112 \times 92$ pixels, with 256 gray levels per pixel. Images of the individuals have been taken varying light intensity, facial expressions (open/closed eyes, smiling/not smiling) and facial details (glasses/no glasses). Images are in PGM format and were taken against a dark homogeneous background, with tilt and rotation up to $20^{\circ}$ and scale variation up to $10 \%$. Sample face images of a person are shown in figure 1 .
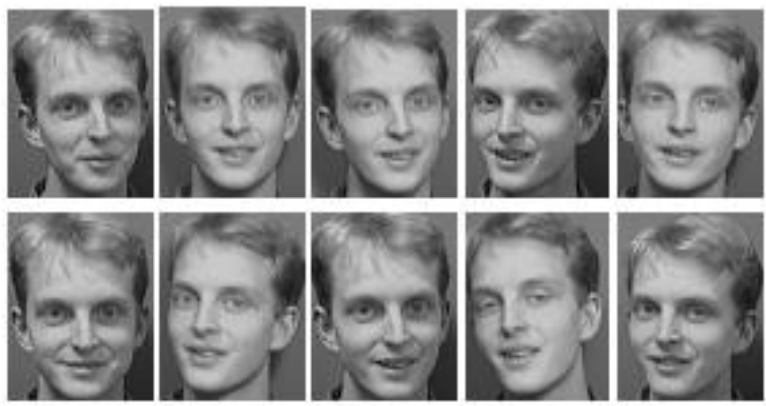

Fig 1: Sample images of a subject from the AT\&T

\section{1 $1^{\text {st }}$ Experiment}

In the first experiment, 200 images of 40 different persons have taken. Each person has 5 images. After training process, different levels of noise have been added with the 200 images and then taken as test images. Next, correctly classified images are counted and listed in a table. In addition, the error curves are given in experimental results.

\section{$2.22^{\text {nd }}$ Experiment}

In this experiment, 5 images of an individual is kept for training and another 5 images of the same individual are kept for testing. Thus, the training data set contains 200 images of 40 different persons. Rest of the 200 images are kept in testing data set.

\section{$2.33^{\text {rd }}$ Experiment}

In this experiment, 200 images of 40 different persons are taken for training. The remaining 200 images are added by different levels of noise and are taken as testing data set.

\section{Program Execution and Performance Testing}

\section{1 $1^{\text {st }}$ Experiment Result}

The eigenfaces corresponding to 10 largest eigenvalues are shown in figure 2. Figure 3 shows the eigenvalue plotted in descending form and eigenvalue spectrum in normalized form and how much variance the first $n$ vectors account for.
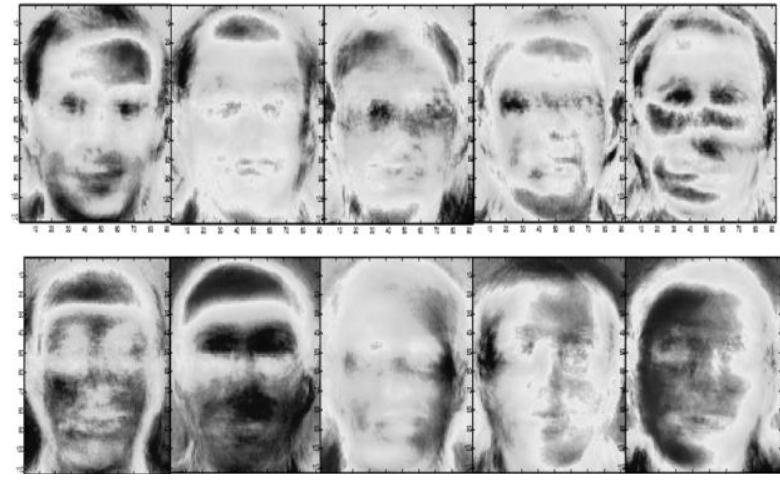

Fig 2: Eigen faces corresponding to 10 largest eigenvalues
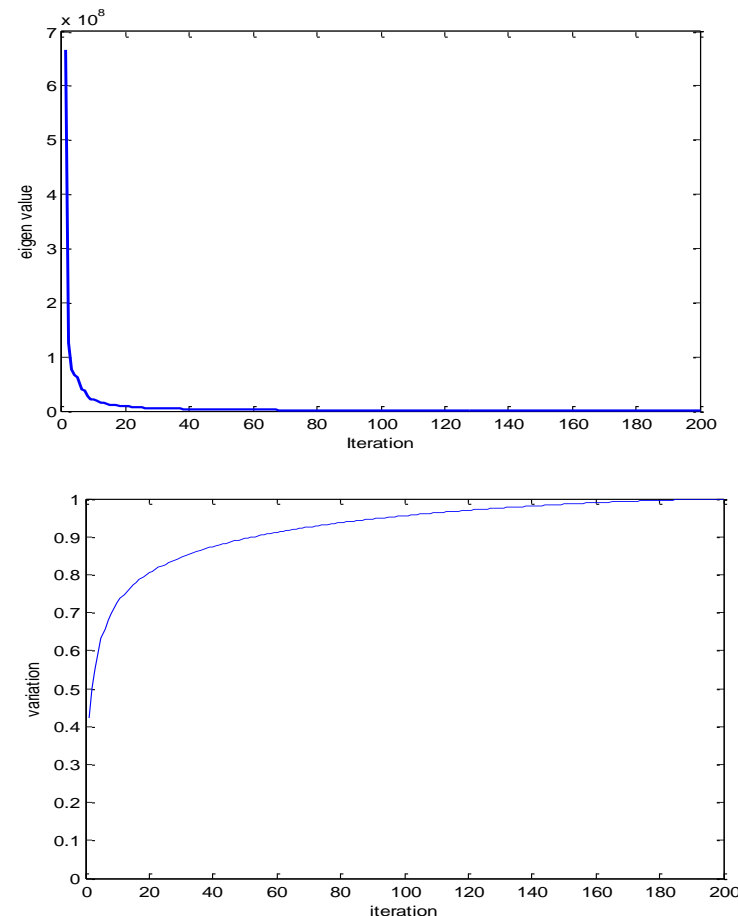

Figure 3. eigenvalue spectrum

The error vs iteration curve for training and testing is shown in figure 4. Here no. of PCs $=80$, no. of hidden neurons $=120$ and noise is zero.

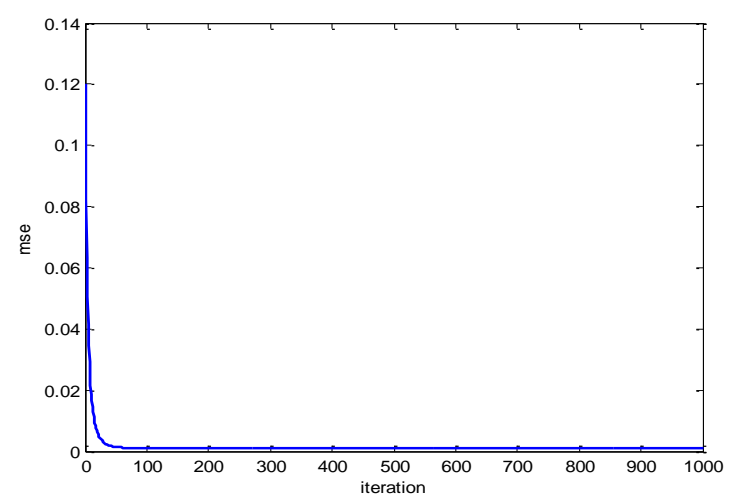

Figure 4(a) Error vs. iteration curves for training 


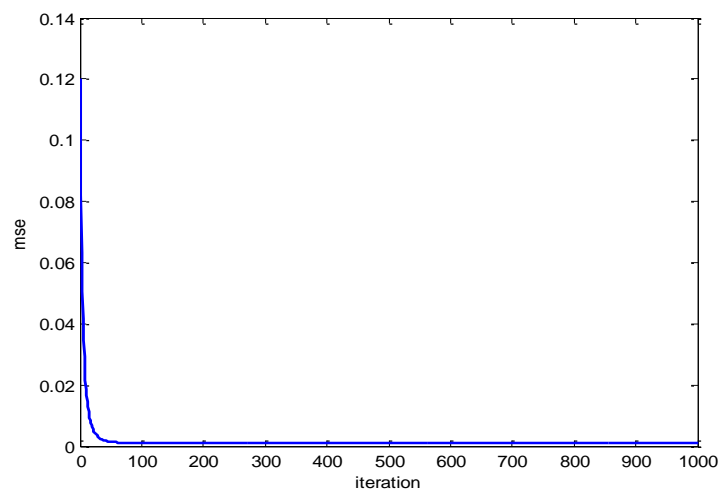

Figure 4(b): Error vs iteration curves for testing

The error vs iteration curve for training and testing is shown in figure 5 where no. of PCs $=80$, no. of hidden neurons $=120$ and noise is zero mean with variance 0.002 .

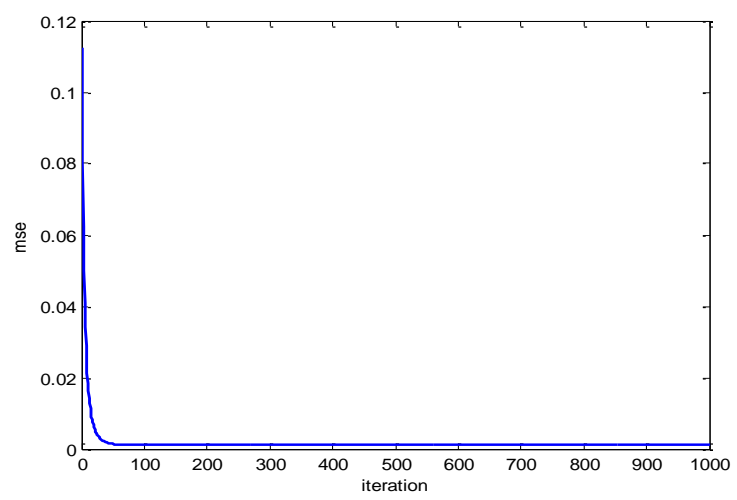

Figure 5 (a): Error vs. Iteration curves for training

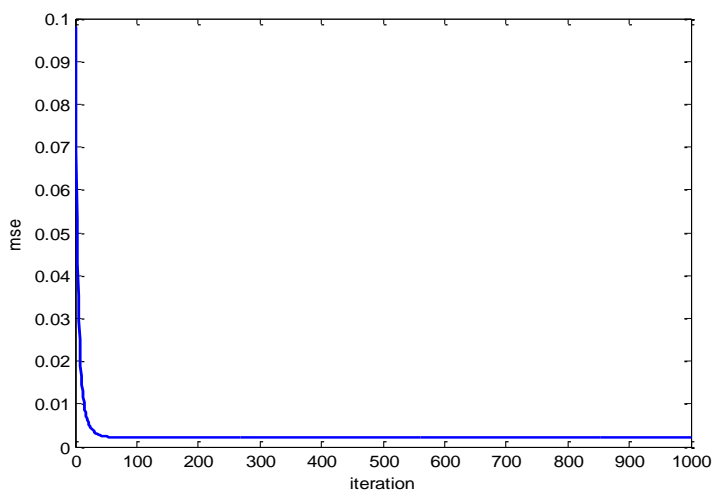

Figure 5 (b): Error vs. Iteration curves for testing

The error vs iteration curve for training and testing is shown in figure.6. Here no. of PCs $=80$, no. of hidden neurons $=120$ and noise is zero mean with variance 0.008

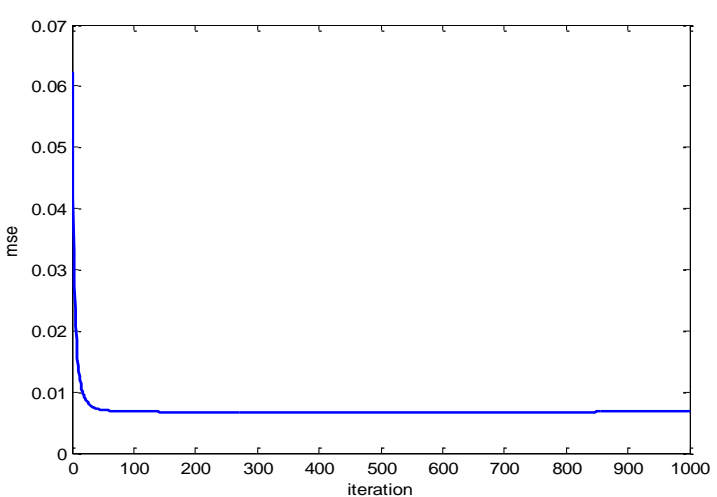

Figure 6 (a): Error vs iteration curves for training

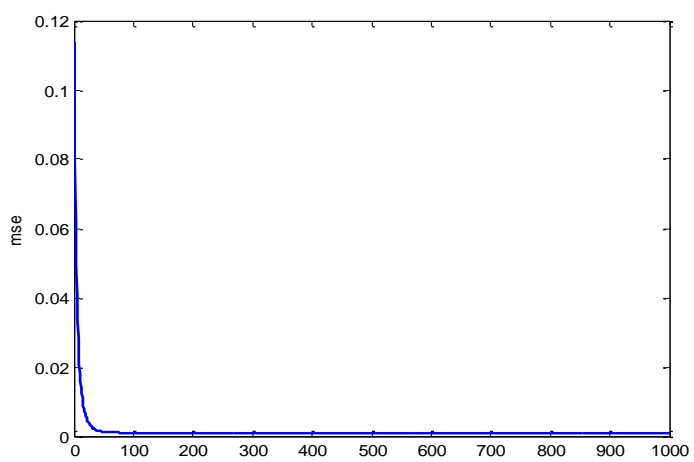

Figure 6 (b): Error vs iteration curves for testing

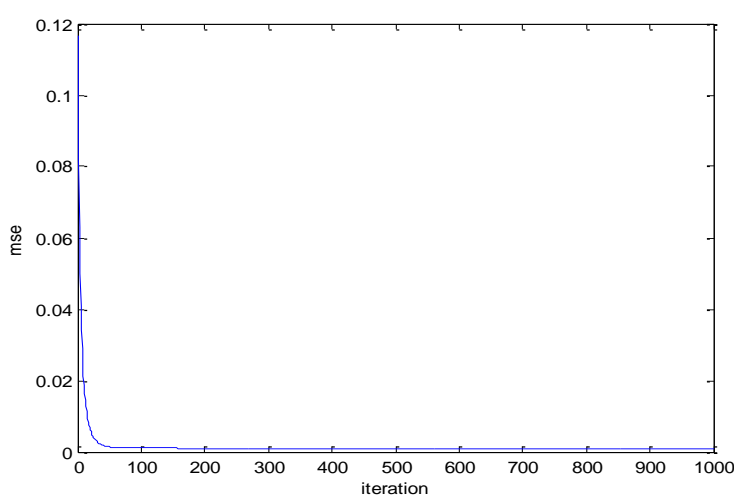

Figure 7 (a): Error vs iteration curves for training

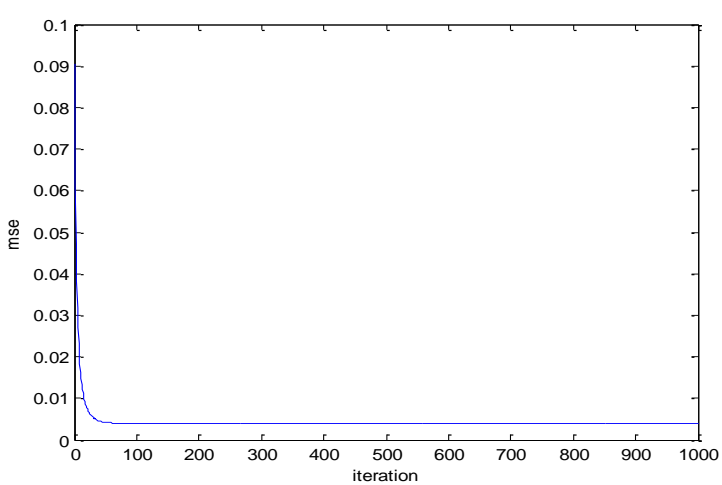

Figure 7 (b): Error vs. iteration curves for testing 
.Network performance for $1^{\text {st }}$ experiment is given below:

Table 1: Network performance for different noise level

\begin{tabular}{|c|c|c|c|c|c|c|}
\hline \multirow[t]{2}{*}{ No. of PCs } & \multirow{2}{*}{$\begin{array}{c}\text { No. of hidden } \\
\text { neurons }\end{array}$} & \multicolumn{2}{|c|}{ Noise } & \multirow[t]{2}{*}{ Recognition } & \multirow{2}{*}{$\begin{array}{c}\text { Recognition rate } \\
(\%)\end{array}$} & \multirow[t]{2}{*}{ Error rate } \\
\hline & & mean & Variance & & & \\
\hline \multirow[t]{9}{*}{80} & \multirow[t]{9}{*}{120} & 0 & 0 & 193 & 96.5 & 0.035 \\
\hline & & 0 & 0.002 & 182 & 91 & 0.09 \\
\hline & & 0 & 0.004 & 162 & 81 & 0.19 \\
\hline & & 0 & 0.006 & 140 & 70 & 0.3 \\
\hline & & 0 & 0.008 & 135 & 67.5 & 0.325 \\
\hline & & 0 & 0.01 & 107 & 53.5 & 0.465 \\
\hline & & 0.01 & 0.003 & 133 & 66.5 & 0.335 \\
\hline & & 0.01 & 0.005 & 123 & 61.5 & 0.385 \\
\hline & & 0.01 & 0.007 & 104 & 52 & 0.48 \\
\hline
\end{tabular}

\section{$3.22^{\text {nd }}$ Experiment Result}

The error vs iteration curve for training and testing is shown in figure 7. Here no. of PCs $=80$ and no. of hidden neurons $=120$. Network performance is observed after performing this experiment for different number of PCs and hidden neurons. Here it is tabulated below in table 2:

Table 2: Network performance for different no. of PCs

\begin{tabular}{|c|c|c|c|c|}
\hline No. of PCs & $\begin{array}{c}\text { No. of hidden } \\
\text { neurons }\end{array}$ & Recognition & $\begin{array}{c}\text { Recognition rate } \\
(\%)\end{array}$ & Error rate \\
\hline 10 & 120 & 148 & 74 & 0.26 \\
\hline 30 & 120 & 160 & 80 & 0.2 \\
\hline 50 & 120 & 155 & 77.5 & 0.225 \\
\hline 70 & 120 & 160 & 80 & 0.19 \\
\hline 90 & 120 & 162 & 82 & 0.18 \\
\hline 100 & 120 & 164 & & \\
\hline
\end{tabular}

Table 3: Network performance for different no. of Nurons

\begin{tabular}{|c|c|c|c|c|}
\hline No. of PCs & $\begin{array}{c}\text { No. of hidden } \\
\text { neurons }\end{array}$ & Recognition & $\begin{array}{c}\text { Recognition rate } \\
(\%)\end{array}$ & Error rate \\
\hline 10 & 60 & 139 & 69.5 & 0.305 \\
\hline 30 & 80 & 143 & 71.5 & 0.285 \\
\hline 50 & 100 & 148 & 74 & 0.26 \\
\hline 70 & 120 & 164 & 82 & 0.18 \\
\hline 80 & 120 & 169 & 84.5 & 0.155 \\
\hline 90 & 140 & 166 & 83 & 0.17 \\
\hline 100 & 140 & 170 & 85 & 0.15 \\
\hline
\end{tabular}

Best network performance is found for $\mathrm{PC}=80$, hidden neuron $=120$ and $P C=100$, hidden neuron $=140$.

\section{$3.33^{\text {rd }}$ Experiment Result}

The error vs iteration curve for training and testing is shown in figure. 8 . Here no. of PCs $=80$, no. of hidden neurons $=120$ and noise is zero mean with variance .002 


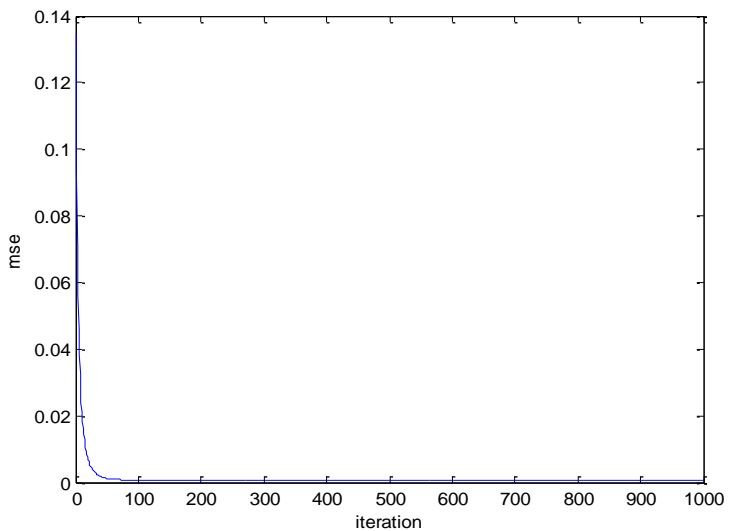

Figure 8 (a). Error vs. iteration curves for training

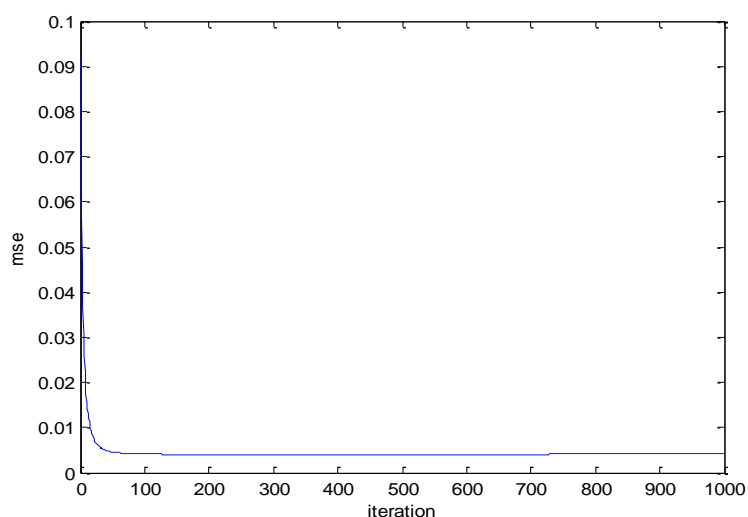

Figure 8 (b). Error vs iteration curves for testing

This experiment is performed for different noise level and then network performance is observed. Table 4. Shows the network performance of $3^{\text {rd }}$ experiment.
The error vs iteration curve for training and testing is shown in figure.9. Here no. of PCs $=80$, no. of hidden neuron $=120$ and noise is zero mean with variance 0.008 .

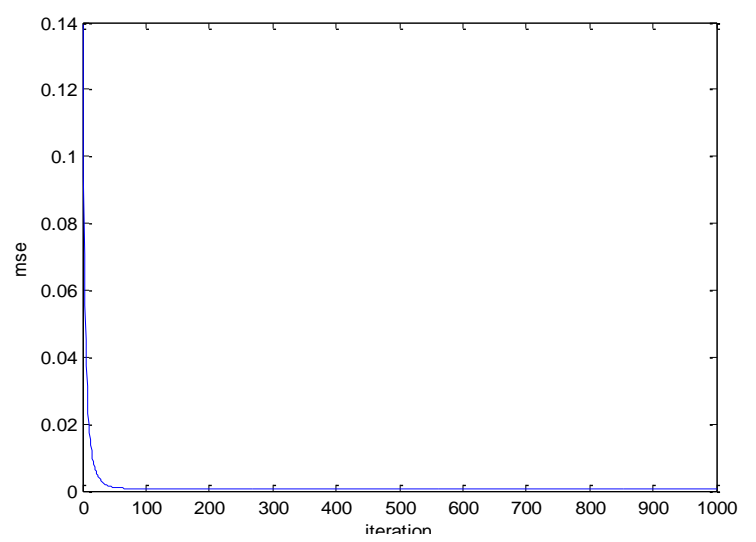

Figure 9 (a). Error vs. iteration curves for training

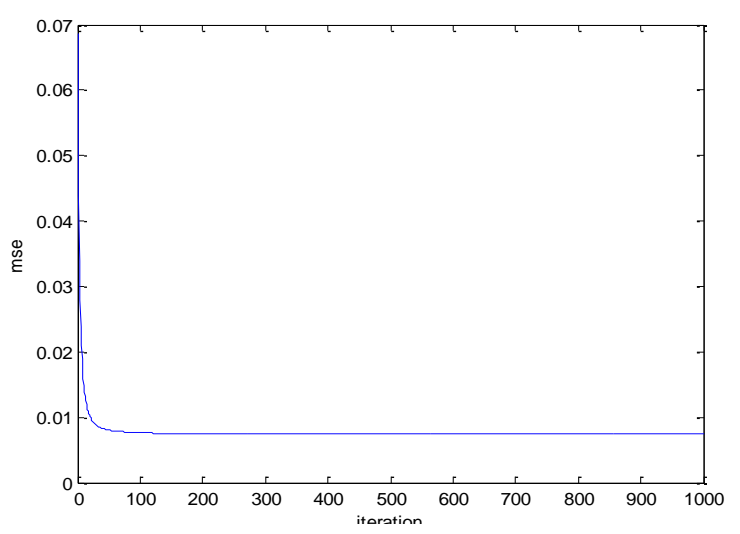

Figure 9 (b). Error vs. iteration curves for testing

Table 4: network performance for different noise level

\begin{tabular}{|c|c|c|c|c|c|c|}
\hline \multirow[t]{2}{*}{ No. of PCs } & \multirow{2}{*}{$\begin{array}{l}\text { No. of hidden } \\
\text { neurons }\end{array}$} & \multicolumn{2}{|c|}{ Noise } & \multirow[t]{2}{*}{ Recognition } & \multirow{2}{*}{$\begin{array}{c}\text { Recognition rate } \\
(\%)\end{array}$} & \multirow[t]{2}{*}{ Error rate } \\
\hline & & Mean & variance & & & \\
\hline \multirow[t]{6}{*}{80} & \multirow[t]{6}{*}{120} & 0 & 0.002 & 156 & 78 & 0.22 \\
\hline & & 0.001 & 0.002 & 156 & 78 & 0.22 \\
\hline & & 0 & 0.004 & 139 & 69.5 & 0.305 \\
\hline & & 0.001 & 0.004 & 138 & 69 & 0.31 \\
\hline & & 0 & 0.006 & 115 & 57.5 & 0.425 \\
\hline & & 0 & 0.008 & 103 & 51.5 & 0.485 \\
\hline
\end{tabular}




\section{Conclusion}

In this paper, an effective method of face recognition technique has been presented. Image processing algorithms based on Radial basis function network has been used in this paper. This paper provides the application of PCA for feature extraction and RBF neural network for classification of face images as a classifier in the context of face recognition technology. It shows the proposed method performed pleasingly for all of the tested application domains. Even, this combination achieves a recognition rate of $96.5 \%$ with zero noise level. However, we have found some limitations too. Recognition rate falls badly as the noise level starts to increase. Therefore, researchers have scopes of works to minimize the noise effect. Introducing filtering networks and combining multi-type methods for feature extraction may increase the performance further.

\section{References}

[1] Meng Joo Er, Shiqian Wu, Juwei Lu, Hock Lye Toh, "Face Recognition With Radial Basis Function (RBF) Neural Networks", IEEE transactions on neural networks, vol. 13, no. 3, may 2002.

[2] V. Radha, N. Nallammal "Neural Network Based Face Recognition Using RBFN Classifier", Proceedings of the World Congress on Engineering and Computer Science 2011 Vol I WCECS 2011, October 19-21, 2011, San Francisco, USA.

[3] L. Wang , X. Wang and J. Feng "On image matrix based feature extraction algorithms", IEEE Trans. Syst., Man, Cybern. B, Cybern., vol. 36, no. 1, pp.194 -197 2006

[4] Suganthy, M. and P. Ramamoorthy, "Principal Component Analysis Based Feature Extraction, Morphological Edge Detection and Localization for Fast Iris Recognition", Journal of Computer Science 8 (9), pp.1428-1433, 2012
[5] Wang, Y., Jiar, Y., Hu, C., \& Turk, M "Face recognition based on kernel radial basis function networks". Asian Conference on Computer Vision, Korea. (2004, January 27-30).

[6] Lindsay I Smith "A tutorial on Principal Components Analysis" February 26, 2002.

[7] Powell, M. J. D, "Radial basis functions for multivariable interpolation:A review" In Algorithms for Approximation, J. C. Mason and M. G. Cox, Eds. Oxford University Press, Oxford, UK, 1987, pp. 143-167

[8] L.N.M.Tawfiq ,Q.H. Eqhaar, "ON RADIAL BASIS FUNCTION NEURAL NETWORKS"Journal of alqadisiyah for pure science(quarterly). Vol-12, pages-1218

[9] Babu, R.V. Suresh, S. Makur, "A. ROBUST OBJECT TRACKING WITH RADIAL BASIS FUNCTION NETWORKS” IEEE international conference on Acoustics, Speech and Signal processing, 2007. Volume: 1, Page(s): I-937 - I-940.

[10] Aleix M. MartõÂnez, Avinash C. Kak, "A. ROBUST OBJECT TRACKING WITH RADIAL BASIS FUNCTION NETWORKS" IEEE Trans. Pattern Analysis and Machine Intelligence, vol. 23, no. 2, pp. 228-233, February 2001

[11] Tiantian Xie, Hao $\mathrm{Yu}$ and Bogdan Wilamowski, "Comparison between Traditional Neural Networks and Radial Basis Function Networks"Industrial Electronics(ISIE), 2011 IEEE International Symposium on, pp. 1194-1199, Date of conference 27-30 June, 2011

[12] M. Turk, A. Pentland, "Eigen faces for Recognition", Journal of Cognitive Neuroscience, Vol. 3, No. 1, 1991,pp. 71-86 